\title{
INTRODUCCIÓN AL MODELO TOPIC MAPS (ISO/IEC13250:2003)
}

\section{Maria Jesús Colmenero Ruiz}

\section{Resumen}

Se realiza una revisión del estándar ISO/IEC 13250:2003 Topic Maps, resaltando sus posibilidades en el ámbito documental. Tras describir su evolución histórica y la situación actual se desarrollan los conceptos que componen este modelo, los tres elementos básicos (topic, association, y occurrence) y dos más (scope y public subject), analizando sus posibilidades para la organización del conocimiento, como estructura de navegación semántica y para la interoperabilidad. Se analizan los elementos más interesantes de la especificación XTM 1.0 y, por último, se detallan algunos de los entornos en los que está siendo aplicado.

Palabras clave

Topic Maps; ISO 13250:2003; XTM; aplicación XML;

\section{INTRODUCTION TO TOPIC MAPS MODEL(ISO/IEC13250:2003)}

\begin{abstract}
It was made a revision of standard ISO/IEC 13250:2003 Topic Maps, standing out its possibilities in the documentary scope. After describes its historical evolution and the present situation the concepts that compose this model, the three basic elements (topic, association, and occurrence) are developed and two more (scope and public subject), analyzing its possibilities for the organization of the knowledge, like semantic structure of browsingn and for the interoperability. The most interesting elements of specification XTM 1,0 are analyzed and, finally, some of the surroundings are detailed in which it is being applied.
\end{abstract}

Keywords

Topic Maps; ISO 13250:2003; XTM; aplicación XML; 


\section{INTRODUCCIÓN}

La publicación del modelo Topic Maps ${ }^{1}$ como estándar por la International Standard Organization (ISO) en el año 2000 y su posterior adaptación al lenguaje XML mediante la especificación XTM, para una mejor incorporación de éste a la Web, ha ido despertando un interés creciente.

A pesar de que el modelo tiene, en un primer acercamiento, un componente principalmente técnico o tecnológico que puede dificultar su comprensión, los presupuestos teóricos que lo sustentan conceptualmente son muy cercanos a los que maneja la Documentación. Al dirigirse sus primeras aplicaciones hacia el desarrollo de sistemas informáticos de Gestión de Conocimiento empresarial y el mantenimiento y gestión de sitios Web, los documentalistas del área comenzaron a prestarle atención, al tiempo que sus impulsores desde el área técnica descubrían la utilidad de los vocabularios controlados como punto de partida idóneo para la construcción de Topic Maps.

Los especialistas en Documentación han comenzado a utilizarlo, precisamente, para adaptar los vocabularios controlados para su uso en web. Resulta de especial interés para dar un paso adelante en la evolución de los tesauros, permitiéndoles adquirir un carácter más asociativo, mejorar su papel de representación del conocimiento de un dominio, acercarse al lenguaje del usuario e integrarse en la Web para la organización y recuperación documental. Por supuesto, su integración como sistema de organización interno en bibliotecas digitales, otra posible aplicación ya explorada, es derivación directa de su utilización previa en la gestión de grandes sitios web.

La intención de este artículo es realizar una revisión de este estándar resaltando sus similitudes con algunos instrumentos tradicionales de la Documentación y sus posibilidades de aplicación documental en el entorno digital.

\section{EL ESTÁNDAR TOPIC MAPS}

Los inicios del estándar Topic Maps se localizan en los trabajos que el llamado Grupo de Davenport, surgido de un consorcio de empresas, comenzó en 1991 con la intención de desarrollar un estándar para la documentación técnica de software. Dividido en dos subgrupos en

\footnotetext{
${ }^{1}$ Al no existir una traducción de este término en nuestro idioma seguiremos la costumbre observada en la escasa literatura que puede consultarse en español, manteniendo el término en el idioma original, el inglés.
} 
1993, uno de ellos se centró en definir una DTD (Document Type Declaration) ${ }^{2}$ para el contenido de los manuales, que dio como resultado la DTD DocBook ${ }^{3}$; el otro subgrupo, Conventions for the Application of HyTime $(\mathrm{CApH})$, tenía como objetivo desarrollar un índice común a partir de distintas fuentes de documentación sobre X-Windows que sirviera como base a otros índices derivados. Esta tarea, que en principio les pareció que no revestiría grandes dificultades, resultó más compleja de lo esperado.

Los esfuerzos de este grupo se centraron en entender la semántica esencial de los índices analíticos de los libros para que fuera posible captarla en forma adecuada para ser procesada mediante ordenador. En palabras de Steve Newcomb, co-editor del estándar, junto a Michel Biezunski (1996):

Esta primera inspiración [...] fue que los índices, si tenían alguna consistencia, se ajustaban a modelos de la estructura del conocimiento disponible en los materiales que indizaban. Pero los modelos estaban implícitos, iy no había forma de encontrarlos! Si estos modelos podían capturarse formalmente, entonces podrían guiar y facilitar enormemente el proceso de fusión de los índices modelados. Pero ¿cómo expresar estos modelos? ${ }^{4}$.

Una vez entendido, el modelo se generalizó para incluir los equivalentes electrónicos de otros elementos procedentes del ámbito impreso que sirven de ayuda a la navegación: tablas de contenidos, referencias cruzadas, glosarios y tesauros (PEPPER, 1999).

En 1996 el grupo de trabajo de SGML de la ISO aceptó el borrador desarrollado, denominado entonces "Topic Navigation Maps", como nuevo documento de trabajo. Finalmente, en el verano de 1999 fue aceptado como norma y publicada como ISO/IEC 13250:2000 Topic Maps

2 Una DTD es un documento que permite establecer restricciones en los documentos SGML (Standard Generalized Markup Language) y XML (eXtensible Markup Language), para asegurar que éstos ajustan su formato a una determinada estructura. A diferencia de los documentos SGML, los documentos XML no están obligados a ajustarse a las restricciones impuestas por una DTD. Cuando lo hacen se dice que ese documento es válido.

3 Disponible en <http://www.docbook.org/>. DocBook es una DTD para el lenguaje SGML (Standard Generalized Markup Language), el lenguaje de marcas del cual deriva HTML. Aunque se diseñó para documentación del área informática, muchos autores de otros campos la han ido adaptando porque su estructura refleja la idea abstracta que se tiene de un libro.

4 "That first inspiration [...] was that indexes, if they have any self-consistency at all, conform to models of the structure of the knowledge available in the materials that they index. But the models are implicit, and they are nowhere to be found! If such models could be captured formally, then they could guide and greatly facilitate the process of merging modelled indexes together. But how to express such models?". Cit. (PEPPER, 1999). 
(International Organization for Standardization, 2000). Está descrito en lenguaje HyTime (Hypermedia/Time-based Structuring Language) el cual, a su vez, es una aplicación del lenguaje SGML (Standard Generalized Markup Language) para procesar documentos 5 . De forma coloquial se conoce como HyTM. Esta sintaxis de intercambio es muy flexible lo que permite a los usuarios escribir sus propias DTDs y el uso de una gran variedad de estructuras de enlace multimedia definidas mediante HyTime (AHMED, 2003). Sin embargo, el número de personas que dominan este lenguaje no es elevado pues a pesar de su flexibilidad, o a causa de ello, resulta complejo.

A comienzos de 2000 se funda una organización independiente, TopicMaps.Org, con el objetivo de adaptar esta norma a un lenguaje más adecuado para la Web. Basándose en las recomendaciones XML y el estándar para enlaces XLink del W3C, publicó la primera versión de una DTD para expresar Topic Maps en marzo de 2001, conocida como XTM.

La especificación XTM 1.0. (TopicMaps.Org, 2001) fue admitida por la ISO e incorporada al estándar mediante una Enmienda Técnica en octubre de ese mismo año. En mayo de 2002 el Subcomité 34 del JTC1 de la ISO/IEC (Join ISO/ICE Technical Comité SC34) aprobó y publicó la segunda edición de la norma ${ }^{6}$, recogida finalmente por la ISO como ISO/IEC 13250:2003. Topic Maps (International Organization for Standardization, 2003), incorporando en su Anexo C la sintaxis XML.

El modelo intenta proporcionar un esquema de representación de estructuras de conocimiento, en forma de red semántica, y asociarlas con recursos de información, y que incorpore un valor añadido similar al que un índice analítico añade a un libro. Denominado "GPS del universo de información" por Charles F. Goldfarb, el desarrollador de los lenguajes de marcado y del lenguaje SGML (RATH, 2003), los topics maps permiten tanto organizar documentos digitales como navegar a través de la estructura semántica que los conecta entre sí.

La norma especifica no sólo los conceptos que conforman los topic maps sino también el formato de intercambio estándar de topic maps entre diferentes aplicaciones.

\footnotetext{
${ }^{5}$ Ambos lenguajes son igualmente estándares aceptados por la ISO: ISO 8879:1986. Information processing. Text and office systems. Standard Generalized Markup Language (SGML) e ISO 10744:1997, Information processing. Text and office systems. Hypermedia/Time-based Structuring Language (HyTime).

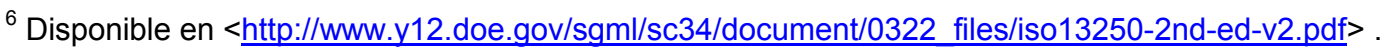


Los topic maps sólo tienen sentido en el medio digital, constituyendo ellos mismos un hiperdocumento, que conecta semánticamente información heterogénea, formando una capa independiente por encima de los recursos, por lo que pueden aplicarse a grandes colecciones de información y en continuo crecimiento.

Básicamente enlazan conceptos mediante asociaciones, en principio sin limitación alguna, lo que los asemeja a otros instrumentos (como tesauros, mapas conceptuales u ontologías, por ejemplo) y permite que sean utilizados para modelar y expresar cualquiera de ellos ${ }^{7}$. En particular, en relación con los instrumentos documentales, esta similitud fue patente desde un principio para sus desarrolladores:

Un Topic Map es funcionalmente equivalente a los índices multidocumentales, glosarios y tesauros ${ }^{8}$. (BIEZUNSKI; HAMON, 1996).

Dado que el modelo básico de las redes semánticas es muy similar al de conceptos y asociaciones de los índices, combinar los dos enfoques puede proporcionar grandes beneficios tanto a la gestión de información como a la gestión del conocimiento, y esto es, precisamente, lo que el nuevo estándar topics maps logra. Añadiendo el eje topic/ocurrence al modelo topicl association, los topic maps proporcionan una forma de "salvar la distancia" existente entre la representación del conocimiento y el campo de la gestión de información ${ }^{9}$. (PEPPER, 2002).

Más recientemente Piotr Kaminsky (2002, p. 85) lo expresa así:

Debido a su rica estructura y peculiar terminología, el metamodelo topic maps es con frecuencia mal entendido por las personas con conocimientos sobre metamodelos más simples, pero parece encajar bien con el punto de vista de los bibliotecarios y otros trabajadores de la información ${ }^{10}$

El subcomité SC34 de la ISO continúa trabajando en la evolución del estándar. Sus esfuerzos se están dirigiendo además en tres direcciones: el desarrollo de un lenguaje de interrogación (Topic

7 La posibilidad de crear modelos individuales distintos entre sí permite cualificar al modelo Topic maps como "metamodelo".

8 "A Topic Map is functionally equivalent to multi-document indexes, glossaries, and thesauri".

9 "Since the basic model of semantic networks is very similar to that of the topics and associations found in indexes, combining the two approaches should provide great benefits in both information management and knowledge management, and this is precisely what the new topic map standard achieves. By adding the topic/occurrence axis to the topic/association model, topic maps provide a means of "bridging the gap", as it were, between knowledge representation and the field of information management".

10 "Due to its rich structure and peculiar terminology, the topic maps metamodel is often misunderstood by people with a background in simpler metamodels, but seems to fit well into the worldview of librarians and other information workers". 
Map Query Language; TMQL, ISO/IEC 18048), un lenguaje de restricción para aquellos casos en los que se necesite un modelo más formalizado (Topic Map Constraint Language; TMCL ISO/IEC 19756) y dos modelos de datos, el Modelo de Referencia (Reference Model; RM) y el Modelo de Aplicación Estándar (Standard Application Model; SAM), que constituyen, en realidad, un modelo en dos capas. El modelo de datos tiene como objetivo servir como guía explicativa para los programadores de software y como cuerpo central para posteriores desarrollos, además de aclarar sus relaciones con otros sistemas de representación del conocimiento como RDF y KIF. También pretende definir lo que ha dado en llamar "sintaxis canónica" (Canonical Topic Map syntax) que permita interpretar correctamente los topic maps escritos en cualquiera de las otras dos sintaxis normativas ${ }^{11}$. En abril de 2003 ha publicado un primer borrador de la versión XTM 1.1 (GARSHOL; MOORE, 2003) que está próxima a su aprobación definitiva.

Por su parte, TopicMaps.Org, tras finalizar la especificación XTM. 1.0, se incorporó a la organización técnica OASIS donde se han formado tres comités técnicos enfocados al desarrollo de los denominados published subjects ${ }^{12}$, cuyo objetivo es proporcionar un identificador estable para definiciones de uso común, de tal forma que, a la hora de fusionar dos topic maps, aquellos topics que tienen el mismo identificador se entiende que se están refiriendo al mismo concepto.

El comité técnico Published Subjects (Published Subjects Technical Committee) está encargado de desarrollar directrices y recomendaciones para su creación, publicación y mantenimiento. El comité técnico Vocabulario XML (XML Vocabulary Technical Committee) está encargado de crear un vocabulario u ontología para el dominio de los estándares y tecnologías XML. El comité técnico Geografía y Lenguas (Geography and languages Technical Committee) está desarrollando un conjunto de published subjects para conceptos geográficos y lingüísticos basados en los códigos definidos por las normas ISO 639 e ISO $3166^{13}$.

11 El subcomité mantiene una página informativa sobre sus actividades. A través de la dirección <http://www.y12.doe.gov/sgml/sc34/document/0323.htm>, que proporciona una guía sobre el desarrollo de la familia de estándares topic maps, pueden localizarse todos los documentos de trabajo. También mantiene un topic map sobre la evolución de los trabajos, que se puede consultar y descargar en formato $\quad \mathrm{XTM}$, en <http://www.ontopia.net/omnigator/models/topicmap complete.jsp?tm=tmstandards.xtm>. Igualmente, mantienen otro sitio Web informativo en < http://www.isotopicmaps.org/>.

12 Este término puede ser traducido como "temas o materias publicados". La intención es que sean públicos y puedan ser utilizados libremente por los autores de topic maps.

13 La información sobre estos comités técnicos puede localizarse en <http://www.oasis-

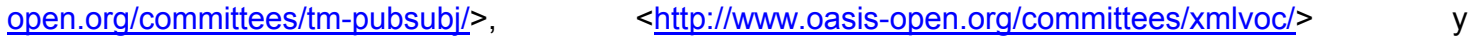
<http://www.oasis-open.org/committees/geolang/> respectivamente. 
Otras personas e instituciones han realizado desarrollos que, si bien no son normativos, suponen avances para la creación de herramientas y para la consolidación del modelo. La Universidad de Bond de Australia ha elaborado el lenguaje AsTMa ${ }^{14}$, una sintaxis que permite crear, mantener, constreñir, limitar y consultar topic maps. Existe también un sistema de notación lineal, LTM (Linear Topic Map Notation), creación de Lars Marius Garshol (2002), que permite expresarlos en una forma textual más clara para las personas. Este autor también ha desarrollado un lenguaje de consulta de topic maps denominado "tolog" (GARSHOL, 2001a). Otros esfuerzos destacables son los realizados por distintas empresas y de grupos de trabajo de software libre en el desarrollo de herramientas que permitan procesarlos; las investigaciones en el campo de su visualización; el diseño de hojas de estilo XSLT de Nikita Ogievetsky (s.d.), que permiten transformar topic maps escritos con diferentes sintaxis, o el lenguaje XFML (eXchangeable Faceted Metadata Language), subconjunto de XTM para intercambio de jerarquías de metadatos facetados por parte de Peter van Dijk (2002), por destacar algunos ejemplos.

La relación de este estándar con las tecnologías que se están intentando establecer para la Web semántica, aunque claras, topa con algunas dificultades. La Web semántica está construida sobre RDF, existiendo problemas de interoperabilidad entre ambos. Analizar y salvar esta distancia ha sido el objetivo de muchos investigadores (FREESE, 2002; GARSHOL, 2001b; GARSHOL, 2003; LACHER; DECKER, 2001; MOORE, 2001; PEPPER, 2002). El desarrollo de OWL sin embargo ha abierto una puerta al engarce, proponiéndose que el lenguaje de constricción de topic maps (TMCL) haga uso de aquél. Ha sido incluido también en la agenda de grupo de trabajo W3C Semantic Web Best Practices and Deployment ${ }^{15}$, creado en marzo de 2004. Bernard Vatant es uno de los mayores impulsores de los lazos de unión entre topic maps y ontologías, habiendo realizado interesantes propuestas en este sentido (VATANT, 2003; VATANT, 2004).

A continuación desarrollaremos los conceptos que componen este modelo, analizando sus posibilidades para la organización del conocimiento, como estructura de navegación semántica y para la interoperabilidad. Nos centraremos en el modelo descrito para la sintaxis XML, es decir, la especificación XTM 1.0, al ser este lenguaje el que se está impulsando en el desarrollo de la Web semántica y, a su vez, el de mayor difusión y menor dificultad de aprendizaje.

\footnotetext{
14 Información disponible en <http:/astma.it.bond.edu.au/>. Esta universidad mantiene una página muy completa sobre topic maps en <http:/topicmaps.bond.edu.au/>.

${ }^{15}$ Disponible en <http://www.w3.org/2001/sw/BestPractices/>.
} 


\section{COMPONENTES DEL MODELO}

Un topic map, como ya se indicó, es un hiperdocumento que forma una red de enlaces semánticos por encima de los recursos de información, asociando tanto los conceptos con los recursos correspondientes como los conceptos entre sí. Así, realizar una búsqueda en la red descrita por el topic map puede asimilarse a la búsqueda en estructuras de conocimiento (HEMRICH; SCHÄFER, 1999).

El núcleo central del modelo definido por el estándar ISO/IEC 13250:2003 Topic Maps está constituido por tres elementos básicos: Topic, Association, y Occurrence. Esta tríada de conceptos fue recogida como el TAO de los topic maps por Steve Pepper (2002), uno de los editores de XTM. Junto a ellos, ampliando el poder expresivo del modelo, se recogen los conceptos de scope, public subject, y facet.

Un topic map se funda sobre el concepto de Topic, el cual constituye la representación material o concreta del Subject, percepción humana abstracta de una realidad. La noción de subject es el punto de partida conceptual sobre el que descansa el modelo, siendo definido en la norma en los siguientes términos.

"En el sentido más amplio, un 'subject' es cualquier cosa, con independencia de si existe o tiene otras características específicas, sobre la cual puede decirse cualquier cosa con cualquier significado". ${ }^{16}$

La nota que acompaña a la definición intenta aclararla indicando que "el corazón invisible de cada topic es el subject que su autor tenía en mente cuando fue creado"17.

Así, el término topic indica el objeto u elemento del topic map que representa al subject al que se está refiriendo, haciéndolo "real" para el sistema. Entre topic y subject se establece una relación biunívoca en la cual un subject es representado por un único topic y viceversa (PEPPER, 2002).

\footnotetext{
16 'In the most generic sense, a 'subject' is any thing whatsoever, regardless of whether it exists or has any other specific characteristics, about which anything whatsoever may be asserted by any means whatsoever".

17 En inglés, las palabras subject y topic son sinónimos cuando actúan como sustantivos, teniendo como significado el de tema, materia. En el modelo topic map el significado de subject está más cercano al de representación mental, concepto, noción o idea. De esta forma, subject y topic constituyen un támden de conceptos semejante al que F. de Saussure desarrolló para el signo lingüístico, significantesignificado.
} 
Al igual que el subject, un topic puede representar cualquier cosa: personas, entidades individuales o colectivas, conceptos, etc.

Se diferencia asimismo entre subjects direccionables y no-direccionables. Los direccionables son aquellos recursos que tienen una existencia en el espacio digital; los no-direccionables son los que existen fuera de este espacio, no pudiendo por tanto ser enlazados directamente, por lo que deben identificarse de forma indirecta mediante un recurso que funciona como subject indicator.

Cada topic es una instancia de una o más clases de topics (denominados también topic types) ${ }^{18}$, que pueden o no indicarse de forma explícita ${ }^{19}$. Los topic types o clase de topic son topics igualmente. Esta relación clase-instancia viene expresada de diferente forma según la sintaxis: HyTime la incluye a través del atributo type del elemento topic link (topic), mientras que la especificación XTM lo realiza mediante el elemento instanceOf. En caso de no definirse ninguno se asume por defecto el definido por el "topic" publised subject $t^{20}$.

El proceso de materialización de los subjects como topics hace posible la asignación de características a éstos últimos. Cada topic puede tener las características siguientes: una denominación (topic name), un o unos ejemplos o descripciones (topic occurrence) y un rol como miembro de una asociación (role). Esta asignación de características se considera válida para un determinado scope o contexto. Dos topics con las mismas características se consideran idénticos, produciendo duplicidad, por lo que uno de ellos se eliminará cuando el topic map sea procesado.

El nombre, legible para los humanos, de un topic se inscribe a través del topic name. Dado que un mismo concepto puede ser designado con una gran variedad de nombres, e incluso ninguno válido, el modelo permite definir nombres normalizados a los topics que sean significativos desde el punto de vista semántico, al mismo tiempo que concede la posibilidad de asociar otros libremente con vistas a su procesamiento por distintas aplicaciones (PEPPER, 2002). Así, un topic puede desde no tener nombre hasta disponer de varios, mediante la asignación de múltiples base name. Sin embargo, cada uno de los distintos base name es válido para un determinado

${ }^{18}$ El término elegido por XTM es clase, mientras que la norma ISO original se decantaba por tipo (type). Ambos son sinónimos en este contexto.

19 Steve Pepper (2002) señala que esta categorización de topics de acuerdo a un tipo corresponde a la categorización inherente al uso de varios índices en un libro (onomástico, de obras, de topónimos) y de la tipografía y otras convenciones para distinguir diferentes tipos de topics. Lo que refleja, una vez más, las raíces documentales del modelo.

${ }^{20}$ Disponible en la dirección http://www.topicmaps.org/xtm/1.0/\#psi-topic. 
scope, no permitiéndose más de uno para el mismo contexto ${ }^{21}$. Adicionalmente, el base name puede incluir formas alternativas o variantes del nombre (variant name) para distintos procesos dependientes de la aplicación, como la forma en que se mostrará el nombre en la pantalla (con cualquier tipo de datos: caracteres, gráficos, etc.) o como será ordenado, por ejemplo ${ }^{22}$.

La derivación principal de que un topic pueda tener una multiplicidad de nombres es que pueden usarse para distintos propósitos. La utilización preferente que de ello se hace, listar sinónimos, variantes dialectales o idiomáticas, es precisamente la que permite incluir las relaciones de equivalencia y de referencia descriptor-no descriptor (usado por-úsese) de un tesauro reuniéndolas todas en torno al concepto. Esta es una de las posibilidades evolutivas del tesauro en el medio digital a las que nos referimos anteriormente.

Una Occurrence es "cualquier información que es especificada como relevante para un subject dado"23 (TOPICMAPS.ORG, 2001). En puridad, son recursos externos de información, enlazados mediante una referencia que sirve para su localización, que aclaran o ejemplifican el significado del topic. Las referencias a recursos se realizan, en XTM, a través de URIs (Uniform Resource Identifiers) según la especificación correspondiente (IETF (Internet Engineering Task Force), 1998).

Estos recursos no se almacenan, habitualmente, en el topic map, lo que implica que el topic map y los recursos que indican las occurrences forman capas separadas; que éstos últimos pueden constituir un conjunto de información de cualquier tipo, formato, o localización; y que los topic maps son transportables siendo posible aplicarlos a diferentes elencos de recursos informativos. Estas cualidades del modelo, consideradas ventajosas en los círculos tecnológicos, en realidad son las mismas que tienen los tesauros respecto a los documentos que indiza: es un instrumento independiente de los recursos, se puede utilizar frente a distintas colecciones documentales y éstas colecciones pueden ser heterogéneas y sus componentes estar radicados en emplazamientos

\footnotetext{
${ }^{21}$ Esta limitación, única en cuanto a las decisiones intelectuales que un autor de topic maps debe hacer, es conocida como "restricción para dar nombre al topic" (topic naming constraint; TNC), estando destinada a facilitar la fusión o unión de dos o más topic maps.

${ }^{22}$ La sintaxis XTM es menos restrictiva respecto a la inclusión de variedades de nombres que la sintaxis HyTime.

23 "any information that is somehow specified as being relevant to a given subject".
} 
distintos. La separación entre recursos y el topic map que los enlaza y organiza tiene la importante ventaja de hacer innecesario el marcado de los documentos con metadatos ${ }^{24}$.

La occurrence puede incluir también información como datos de carácter, lo que es especialmente útil cuando su cantidad es pequeña (por ejemplo, fechas de nacimiento, publicación, coordenadas, definiciones cortas...).

Al igual que con los topics, cada occurrence es instancia de, en este caso sólo de una, una clase de occurrence (denominada también occurrence type ${ }^{25}$ ), que puede o no indicarse de forma explícita, y expresada en XTM mediante el elemento instanceOf. El occurrence type o clase de occurrence es asimimismo un topic. El occurrence type por defecto está definido por el "occurrence" publised subject ${ }^{26}$.

El tercer elemento del núcleo central del modelo topic maps es la Association. Tal y como es definida en TXM, una association es "una relación entre uno o más topics, donde cada uno de ellos juega un rol [role] como miembro [member] de dicha asociación" ${ }^{27}$. El rol que un topic tiene en una asociación, indicando en qué manera participa en ella, es una de las características que puede tener el topic por lo que está condicionado por el contexto (scope) en el que está inscrito.

Esta relación estaría expresada, en forma implícita, por la expresión verbal que uniría los dos topics, asumiendo que éstos representarían los sustantivos de la frase así formada. Por ejemplo, "el sol es una estrella" o "la miel es elaborada por las abejas". El número de topics involucrados en una association no está limitado aunque lo más frecuente es que sean dos (asociaciones binarias) o, en menor grado, tres (asociaciones ternarias).

A semejanza de topics y occurrences, las associations se pueden clasificar en clases o association type. Tal y como se establece para las occurrences, a diferencia de los topics, tan

${ }^{24}$ El que sea innecesario no quiere decir que no sea útil. Sin embargo, es una ventaja por ser aún escaso el número de documentos Web que incluyen metadatos, a pesar de los esfuerzos realizados por impulsar su uso al ser el pilar sobre el que se sustenta la Web semántica.

${ }^{25}$ Este concepto en XTM corresponde con el de occurrence role type en la norma original en HyTime, modificándose su denominación para evitar confusiones con otro concepto distinto. XTM no recoge tampoco el de occurrence role, por considerarse un artefacto de uso del anterior.(PEPPER, 2002).

${ }^{26}$ Disponible en la dirección <http://www.topicmaps.org/xtm/1.0/\#psi-occurrence>.

27 "An association is a relationship between one or more topics, each of which plays a role as a member of that association". 
sólo pueden pertenecer a una clase. E igualmente, se asume por defecto el definido por el "association" publised subject. ${ }^{28}$. La clase o association type es la que concreta, explicita, el verbo que une a los topics y también es un topic.

La capacidad de crear clases de asociaciones, es decir, de crear tipos, hace posible agrupar los topics que tienen la misma relación con un determinado topic, lo que faculta el desarrollo de interfaces intuitivas y amigables para navegar por conjuntos grandes de información (PEPPER, 2002).

La association en el modelo Topic Maps no indica un sentido en el que deba interpretarse. La relación que establece es válida en cualquier caso. Como indica la especificación XTM, de lo que depende es del tipo de relación establecida y del papel que juegan sus miembros en ella: "Cómo marcar una relación es una cuestión de denominaciones, no de dirección"29.

Para poder conocer la función que los topics desempeñan como miembros de una asociación es necesario introducir el concepto de association role. Este concepto debe ser representado mediante un topic, que es el que aclara dicha función. A diferencia de la notación HyTime, XTM no declara un elemento específico para la association role pero lo incorpora a través de la suma de varias partes. Por un lado, una de las tres características del topic es el role o papel jugado en una association. Por el otro, recoge el elemento member, el cual detalla todos los topics que tienen un determinado rol en una asociación, que posee el subelemento roleSpec para definirlo.

En la especificación XTM vienen incluidas dos clases de association, que ya tienen definidos los subjects correspondientes mediante published subjects: la class-instance association (asociación clase-instancia) y la superclass-subclass association (asociación superclase-subclase), con las relaciones entre topics que denotan sus denominaciones.

Estas association nos permiten diferenciar aquellos topics que están funcionando como clases y en qué asociaciones, pues puede ocurrir que un topic sea clase e instancia a la vez, dado que pueden ser instancia de una clase y que éstas son a su vez también topics. La superclass-subclass association permite también construir jerarquías de clases, aplicadas tradicionalmente para realizar taxonomías o clasificaciones de materias. Las relaciones jerárquicas de un tesauro pueden elaborarse en la misma forma. Pueden confeccionarse jerarquías de clases de topics, de clases de associations y de clases de occurrences.

\footnotetext{
${ }^{28}$ Disponible en la dirección <http://www.topicmaps.org/xtm/1.0/\#psi-association>.

29 "The question of how to label a relationship is one of naming, not direction."
} 
Una diferencia fundamental entre estos dos tipos de associations es que la relación superclasesubclase es transitiva, es decir, que las propiedades de la clase superior se van heredando por las subclases inferiores a ella, lo que no ocurre en la relación clase-instancia (RATH, 2003) ${ }^{30}$.

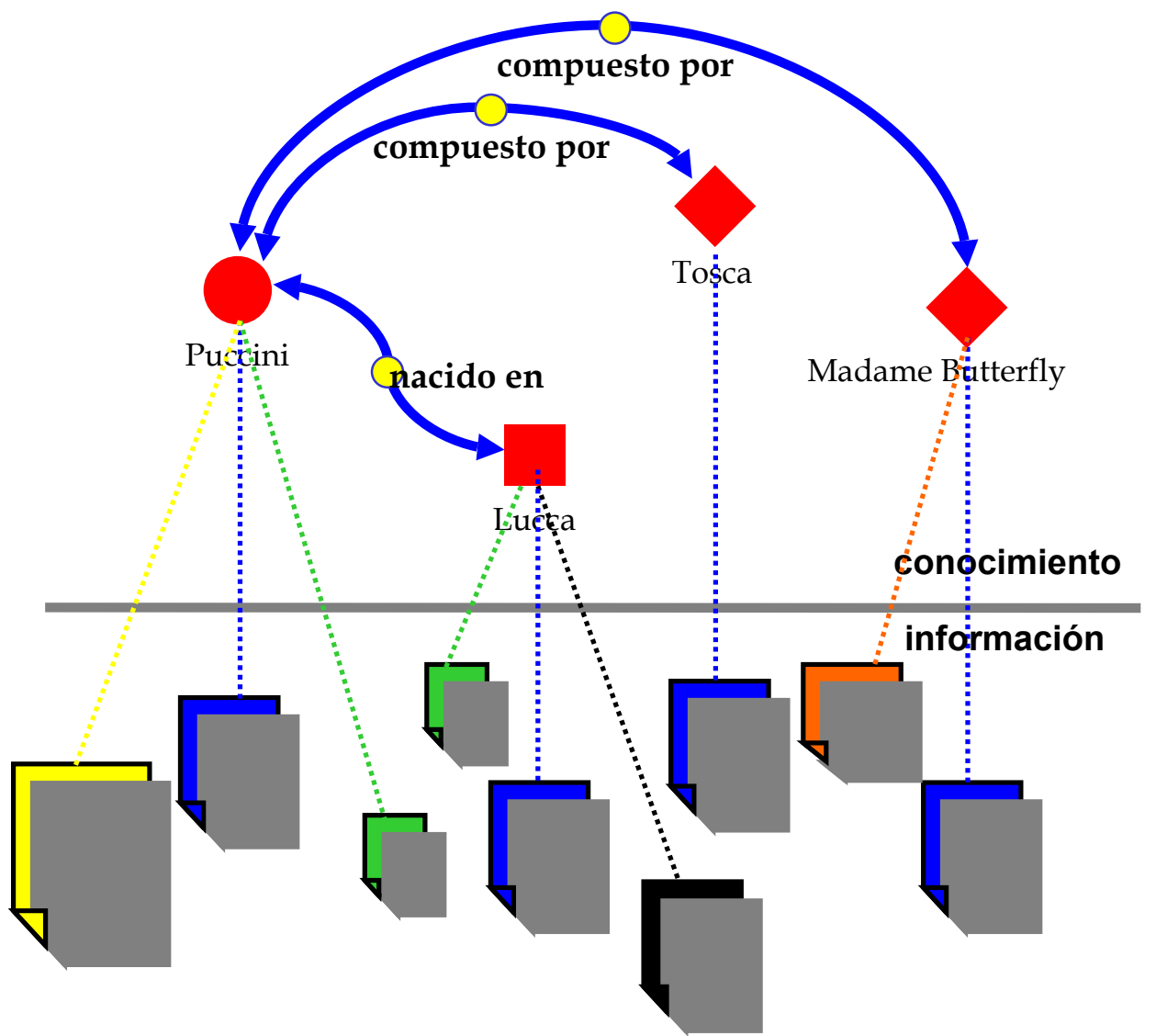

Figura 1. Representación de los principales componentes del modelo Topic maps

[Tomado de (PEPPER, 2004)].

Como ya indicamos, el modelo Topic Maps incorpora otros conceptos, añadidos a los tres centrales, que le dan una potencia mayor. Inicialmente éstos eran tres scope, public subject y facet, pero cuando se desarrolló la especificación XTM el concepto facet no fue incluido. Las facets permitían asignar metadatos, atributos, a los recursos dentro del propio topic map. Steve Pepper (2002) explica su desaparición por haberse convertido en superfluo tras la clarificación realizada entre subjects direccionables y no direccionables ${ }^{31}$, dado que los recursos podían ser

${ }^{30}$ Decir que una relación es transitiva es lo mismo que decir que posee la propiedad matemática de la herencia, lo que implica que una aseveración hecha para una clase es válida igualmente para todas las clases subordinadas a ella.

31 La diferenciación de subjects hizo correr ríos de "tinta" (electrónica) en la lista de distribución topicmapmail (<http://www.infoloom.com/mailman/listinfo/topicmapmail>) al igual que la conveniencia de la desaparición del concepto facet. 
asimismo topics (representando a un subject direccionable) y serles asignadas sus propiedades a través de sus características.

El Scope, como ya se indicó al exponer el concepto topic, constituye el límite de validez de las características asignadas a éste, que puede o no explicitarse, minimizando su ambigüedad. Es decir, el scope es el contexto específico en el que el topic se inscribe al objeto de precisar su significado. El concepto de scope se añadió al modelo topic map para atender al hecho de que raramente hay una única visión del mundo, debido a razones diversas (RATH, 2003): el idioma o dialecto de una comunidad, los derechos de acceso y otra información contextual (por ejemplo, diferentes niveles de destreza o intereses del usuario, o distintas acepciones de una misma palabra (homónimos), a veces incluso contrapuestas).

Existen, como en casos anteriores, diferencias en la forma de incorporar este concepto en la norma ISO 13250 y en XTM. En la primera el scope se define en términos de themes, que son topics también. La segunda obvia el término, refiriéndose a este concepto como "scoping topic" (topic que determina el scope $)^{32}$.

Como son las características del topic las están condicionadas por el scope si éste se aplica y no el propio topic, las posibilidades de uso son extensas. Se pueden destacar algunas, clasificándolas por la característica del topic para la que es empleada (PEPPER; GRØNMO, 2001). En caso de ser utilizado en el nombre, los topics que hacen la función de scope se pueden agrupar en clases según el lenguaje natural que use, la norma aplicada, determinar distintas clases de denominaciones (autoridad, coloquial, mote, pseudónimo) o incluso espacios de tiempo (periodos históricos, calendarios, horarios). Una aplicación, interesante en algunos casos, de esta característica es la posibilidad de etiquetar las clases de asociaciones, permitiendo dar un "sentido de lectura" a la asociación. Ejemplos de uso sobre las occurrences son la gestión de niveles de acceso, subdominios, localización del recurso, comentarios y, de nuevo, el lenguaje que utiliza. Similares son también las aplicaciones derivadas de su uso sobre la función (role) ejercida en las relaciones (association).

Como puede deducirse, la principal consecuencia del uso de scope es que facilita la creación de subconjuntos clasificados de topics, lo que resulta de gran utilidad a la hora de aplicar sistemas de consulta (mediante filtrado) o selección en los interfaces de usuario, mejorando la navegación. Sin embargo, dada la complejidad del concepto por su aplicación a varios elementos

\footnotetext{
32 (PEPPER; GRØNMO, 2001) efectúan un análisis extenso y detallado de este concepto, tanto de su uso y significación como de su tratamiento en ambas sintaxis.
} 
y las implicaciones que ello conlleva, parece necesario profundizar en su desarrollo para conseguir mejores resultados, proporcionándole algún tipo de estructura interna (PEPPER; GRØNMO, 2001).

El último concepto que conforma el modelo es el de Public Subject. Su necesidad deriva de la posibilidad proporcionada de fusionar topic maps entre sí, pudiéndose dar la circunstancia de que un subject no direccionable sea representado por más de un topic, situación habitual si, por ejemplo, provienen de idiomas o dominios distintos ("España" y "Spain" pueden ser topics distintos que están refiriéndose a la misma entidad). La forma de poder establecer la identidad entre estos topic aparentemente distintos es a través del public subject (o public subject indicator; PSI), el cual no es más que un recurso electrónico que identifica el subject con la menor ambigüedad posible. El topic debe enlazarlo indicando su referencia mediante el elemento subjectIdentity. Puesto que la utilización de este elemento es opcional, no existen garantías de que la unión será perfecta y que no resultará en varios topics para el mismo concepto ${ }^{33}$. Si están identificados y los topics son iguales, el resultado de la fusión será uno que recoja sus características combinadas.

Vimos ya que para establecer a qué concepto estaba aludiendo un subject no direccionable podía enlazarse un recurso externo, oficial o no, o incluso realizar una definición dentro del propio topic map, que hace la función de subject indicator. Un public subject es, en consecuencia, un subject indicator que se presenta como documento publicado y mantenido en una dirección comunicada, con el fin de proporcionarle una garantía de credibilidad, autoridad u oficialidad. Pueden ser creados por cualquier persona u organismo, con mayor o menor peso específico, para su uso público con el objetivo último de facilitar el intercambio, la unión de topic maps y su transportabilidad ${ }^{34}$.

Para completar la descripción de este estándar nos fijaremos a continuación en la estructura de la DTD para la sintaxis XML, XTM 1.0 Topic Maps, resaltando aquéllos elementos más relacionados con el esqueleto conceptual del modelo que con su procesamiento informático. La

\footnotetext{
${ }^{33}$ Una solución, no completa y semejante en sus objetivos, la proporciona el topic naming constraint ya citado.

${ }^{34}$ Para dar estabilidad a los PSI y establecer unas directrices de elaboración se creó el Comité técnico Published Subjects en el organismo OASIS al que hicimos referencia con mayor amplitud en el apartado anterior.
} 
versión completa original puede consultarse y enlazarse como DTD externa en $<\underline{\text { http://www.topicMaps.org/xtm/1.0/ }}>^{35}$.

Como apoyo adicional se incluye una tabla (Figura 2) con el significado de los símbolos y códigos que se utilizan en las DTDs y, por tanto, en esta especificación.

\begin{tabular}{|c|c|}
\hline SIMBOLO & SIGNIFICADO \\
\hline$<!$ ELEMENT & Definición de elemento \\
\hline$<!$ ATTLIST & Definición de atributos \\
\hline 0 & Agrupan subetiquetas \\
\hline | & $\begin{array}{l}\text { Barra vertical: sólo puede usarse uno de los elementos situados entre el } \\
\text { paréntesis. }\end{array}$ \\
\hline , & Coma: los elementos deben ser usados en el orden que se establece. \\
\hline+ & Suma: el elemento debe aparecer, como mínimo, una vez \\
\hline$?$ & Interrogación: elemento opcional. Si se utiliza sólo puede hacerse una vez \\
\hline \multirow[t]{2}{*}{ * } & $\begin{array}{l}\text { Asterisco: elemento opcional. Si se utiliza puede hacerse tantas veces como } \\
\text { se desee. }\end{array}$ \\
\hline & Si no se incluye ninguna notación el elemento debe aparecer una sola vez. \\
\hline CDATA & El elemento puede contener cualquier cadena de caracteres \\
\hline \#FIXED & El contenido del elemento está preestablecido \\
\hline \#IMPLIED & El contenido del elemento es opcional \\
\hline ID & Identificador \\
\hline$<!--\quad-->$ & Delimitadores de comentarios y aclaraciones \\
\hline
\end{tabular}

Figura 2. Símbolos y códigos utilizados en las DTD.

${ }^{35}$ Desde la página http://www.doteine.uc3m.es puede enlazarse a una traducción al español de la especificación, con los comentarios de la DTD traducidos para una mejor comprensión. Se sigue así la línea iniciada por los franceses, cuya traducción de la especificación mantiene las etiquetas en el idioma original. (<http://xmlfr.org/topicmaps.org/xtm/1.0/\#ODT>). 
El elemento raíz de esta DTD es el elemento o etiqueta topicMap. Éste puede incluir sólo uno de los subelementos (o subetiquetas) topic, association, mergemap, como indica la barra vertical, aunque pueden repetirse en cualquier orden cuantas veces se desee (indicado por el asterisco tras el paréntesis).

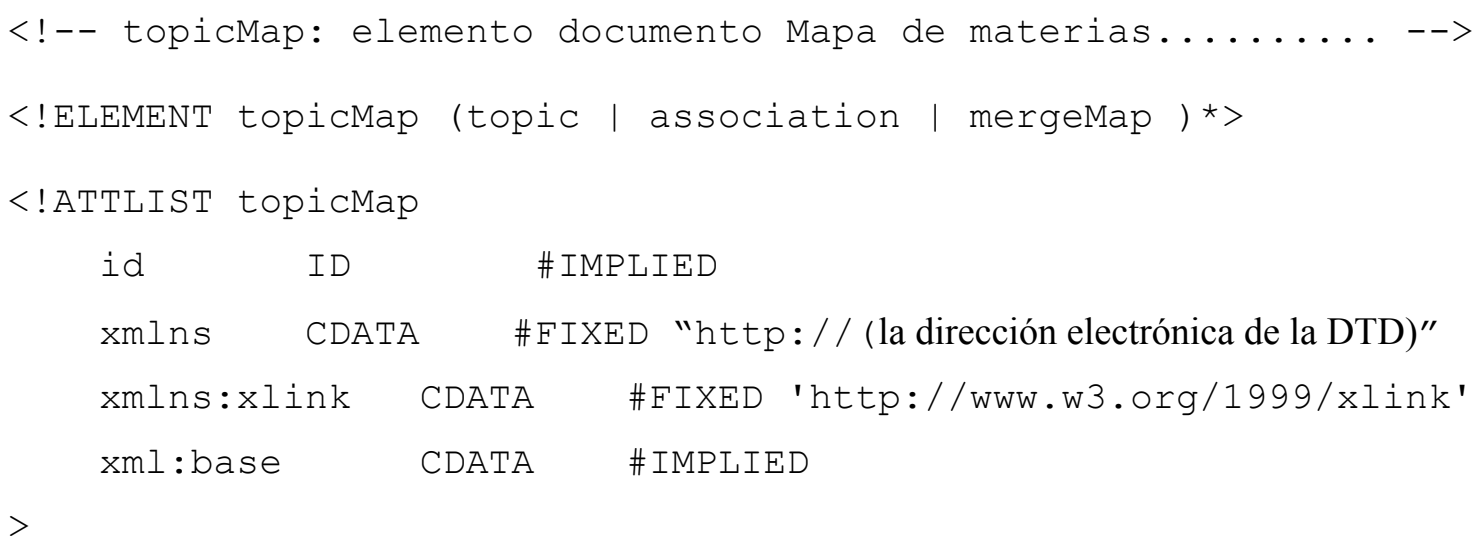

Un ejemplo de documento XML que exprese un Topic Map, que como todos los documentos XML debe comenzar con una cabecera que especifique la versión de XML, tendría descrito este elemento en la siguiente forma ${ }^{36}$ :

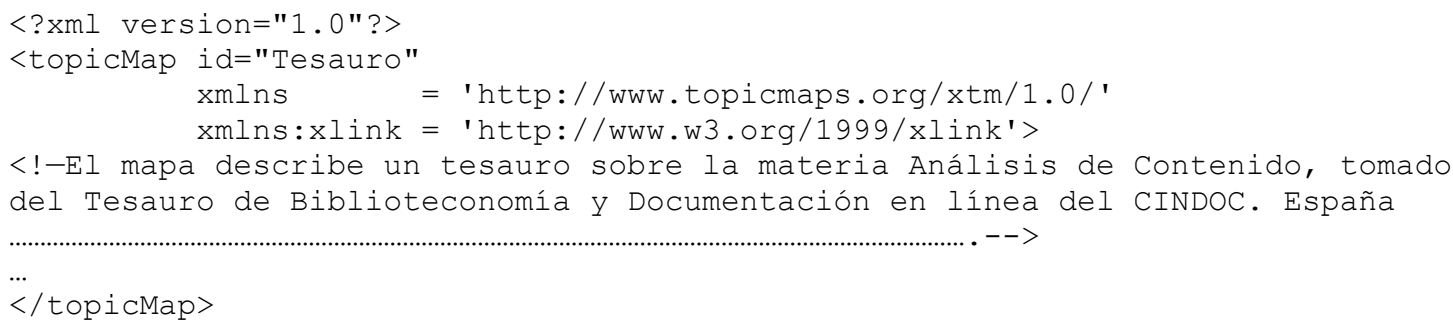

El elemento topic debe estar identificado, internamente, mediante un identificador. En este caso, a diferencia del anterior, el identificador es obligatorio, y único, para poder servir como referencia de enlace desde cualquier otro lugar. Incluye los subelementos instanceof, subjectIdentity, baseName y occurrence:

${ }^{36}$ Este es el único ejemplo que incluiremos para ilustrar como quedarían en el documento XML topic map los elementos que se irán describiendo. Pueden consultarse en (ALTENBURGER, A., 2000) o (MOREIRO GONZÁLEZ, J. A.; SÁNCHEZ CUADRADO, S.; MORATO LARA, J., 2003) los más comunes y en la propia especificación. 
$<$ !- topic: elemento Topic.......................

$<$ ! ELEMENT topic

(instanceof*, subjectIdentity?, (baseName | occurrence)*)>

$<$ !ATTLIST topic

id ID \#REQUIRED

$>$

<!-- instanceof: Apunta a un Topic representando una clase ... -->

$<$ !ELEMENT instanceOf (topicRef | subjectIndicatorRef) >

$<$ !ATTLIST instanceof

id ID \#IMPLIED

$>$

<!-- subjectIdentity: Subject representado por el Topic .... --> $<$ !ELEMENT subjectIdentity

(resourceRef?, (topicRef | subjectIndicatorRef) * ) >

$<$ !ATTLIST subjectIdentity

id ID \#IMPLIED

$>$

<!-- topicRef: Referencia a un elemento Topic............-->

$<$ !ELEMENT topicRef EMPTY >

$<$ !ATTLIST topicRef

id ID \#IMPLIED

xlink:type NMTOKEN \#FIXED 'simple'

xlink:href CDATA \#REQUIRED

$>$

<!-- subjectIndicatorRef: Referencia a un indicador de Subject->

$<$ !ELEMENT subjectIndicatorRef EMPTY >

$<$ !ATTLIST subjectIndicatorRef

id ID \#IMPLIED

xlink:type NMTOKEN \#FIXED 'simple'

xlink:href CDATA \#REQUIRED

$>$ 
Todos los subelementos que componen los elementos instanceof y subjectIdentity, constituyen en definitiva elementos de enlace o referencias, bien internas, bien externas. Son elementos que se van repitiendo a su vez en otros, realizando la misma función (Figura 3). Unos permiten crear el "esqueleto" interno del topic map, mientras que otros enlazan con recursos externos a él.

\begin{tabular}{|l|l|}
\hline & Nomento \\
\hline (topicRef $\mid$ subjectIndicatorRef $)$ & 6 \\
\hline (topicRef $\mid$ subjectIndicatorRef $\mid$ resourceRef $)$ & 5 \\
\hline (resourceRef $\mid$ resourceData $)$ & 2 \\
\hline
\end{tabular}

Figura 3. Repeticiones de elementos en XTM [(MUGNAINI, L., [s.d.]].

Mientras el ID de un topic sirve como representación interna de éste, el nombre de base, que puede indicarse o no dentro del elemento basename, constituye una representación externa, la forma en que se presentará al usuario. Ambas identificaciones pueden diferir (ALTENBURGER, 2000).

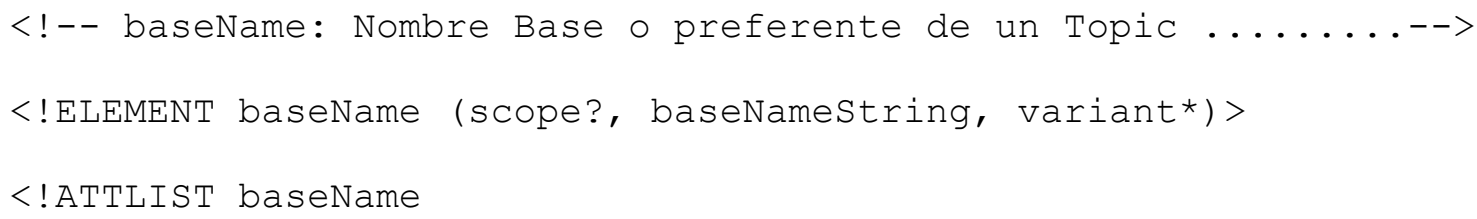

En el elemento baseNameString es donde se recoge el nombre base, que es único. El elemento variant, repetible y con varios subelementos indica sus variantes posibles. En éste último pueden indicarse incluso variantes de variantes (por ejemplo, ortográficas). El elemento scope es opcional pero de un solo uso y compuesto de subelementos que constituyen su referencia (los subelementos que sirven para enlazar responden al mismo concepto y, por tanto, las etiquetas se van repitiendo en los lugares correspondientes).

El subelemento occurrence del elemento topic, está definido como:

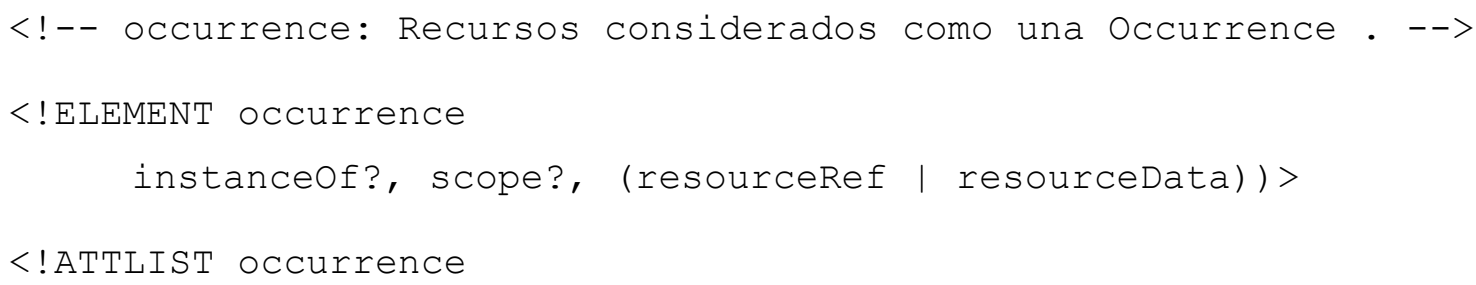


ResourceRef y resourceData permiten enlazar los recursos e incluir metadatos de distinto tipo. Al igual que con los nombres, un topic puede estar enlazado varias ocurrences.

El subelemento association del elemento topicMap puede incorporar los subelementos instanceof, scope y member, debiendo aparecer este último al menos una vez, puesto que define el tipo de participación del topic en la relación.

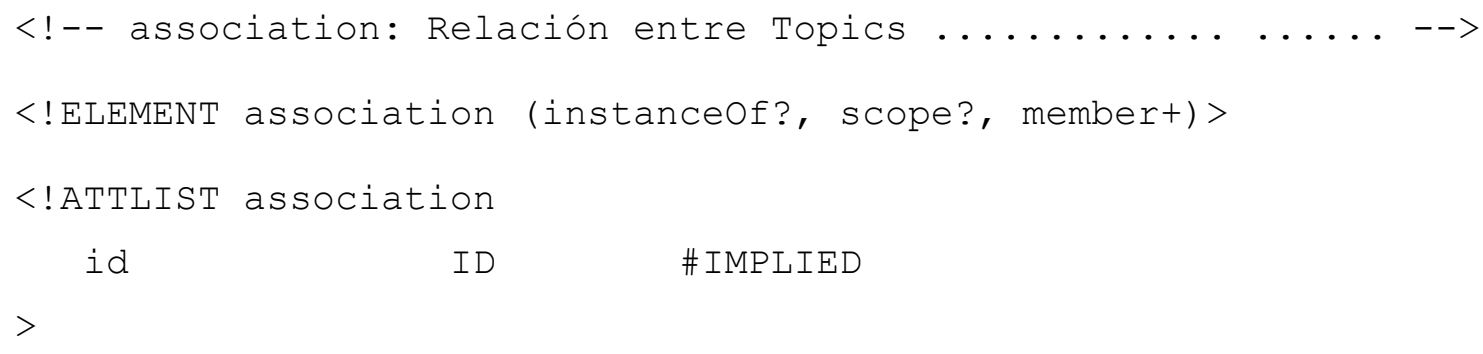

Es de destacar el subelemento roleSpec de member dado que es el que, enlazando con otro topic que lo indica, describe el papel desempeñado por el topic en la relación. Por ejemplo, dada una relación genérico-específico de un tesauro, member apuntaría a un topic identificado como ID="genérico-específico", el tipo de relación, y roleSpec apuntaría a un topic identificado como ID="genérico" o ID="específico", dejando claro cual de los dos términos es el topic considerado.

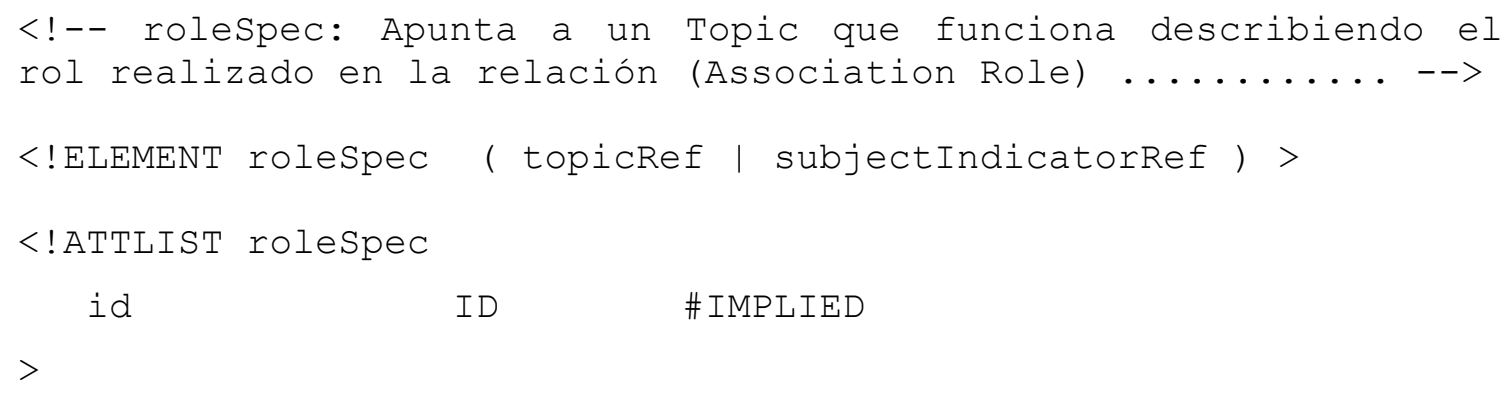

El subelemento mergeMap del elemento topicMap, por último, es el que facilita la unión de mapas haciendo referencia al que quiere incorporarse. Es el mecanismo que permite la actualización, para la incorporación de nuevos recursos o de la estructura de conocimiento del propio mapa. Es posible únicamente a través de una aplicación que procese este elemento.

Como puede observarse, el modelo Topic Map está constituido por un pequeño número de conceptos, lo que le da apariencia de simplicidad. Sin embargo, la combinación de éstos permite 
la descripción de estructuras altamente $\operatorname{complejas}^{37}$, haciéndolo apropiado para su uso en numerosas aplicaciones.

\section{ENTORNOS DE APLICACIÓN}

Las aplicaciones del modelo Topic Map derivan fundamentalmente de dos de sus características principales: formar una capa o nivel independiente de los recursos que organiza, sean éstos documentos con cualquier grado de estructuración o datos, y la facultad de navegar a través de la estructura semántica que conforman y los recursos que enlaza. A éstas se suma la de su reutilizabilidad e interoperabilidad, condición sine qua non para su aplicación en la Web semántica, derivadas del lenguaje XML en que son descritos.

La primera característica posibilita su uso para la organización y representación del conocimiento sobre un dominio específico ${ }^{38}$, asemejándolos en esta forma a las ontologías ${ }^{39}$, por ser capaces de incorporar las características de interés que éstas tienen para la Web semántica. Son considerados como ontologías no refinadas, al no constar de reglas de inferencia o axiomas, pero, por este mismo motivo, son menos costosas de elaborar y adecuadas a los recursos, en su mayoría desestructurados, de la Web. Esto implica que sus aplicaciones posibles son semejantes a las de este tipo de ontologías. Las posibilidades de ampliación a los usos que tienen las ontologías más formales dependen de la viabilidad de los esfuerzos centrados en la compatibilidad con OWL (Ontology Web Language), para incorporar los axiomas que actualmente el modelo no recoge.

La segunda característica es, en sí misma, una aplicación: un sistema de navegación hipertextual, semejante a una segunda generación de sistemas hipertextuales caracterizados por la separación

${ }^{37}$ Esta complejidad, que emerge rápidamente, es la que hace necesario un lenguaje de restricción que limite o constriña el topic map para evitar inconsistencias (GRØNMO, 2000). El primer esquema, que es como se denomina a los lenguajes que permiten limitar la validez de los datos, publicado para XTM fue escrito en XML Schema por Diffuse.org como parte de un proyecto europeo. Sin embargo, la comunidad topic maps, en su afán por alinearse en la Web semántica, ha concentrado sus esfuerzos en que TMCN sea compatible con OWL (Ontology Web Language), lenguaje desarrollado para expresar ontologías en entorno web.

${ }^{38}$ El conocimiento incorporado puede estar basado en un corpus documental, reflejar simplemente el de un sólo documento, como por ejemplo, el expresado por un vocabulario controlado, o recoger el de un experto en el dominio considerado.

${ }^{39}$ La relación entre topic maps y ontologías puede consultarse con más detalle en (OBRST; LIU, 2002). En (FREESE, 2000) y (SIGEL; 2000) se analiza igualmente su uso en estos campos. 
entre enlaces y sistema de navegación y los recursos (KAMEL BOULOS; OUDSARI; ARSON, 2002).

La combinación de ambas características permite aplicaciones multifuncionales, considerando la navegación sólo a través de la estructura del conocimiento expresado y la navegación tanto por la estructura como por los recursos. Los avances en visualización, a través de programas de procesamiento de grafos, están permitiendo, además, la expresión "tridimensional" de la red de conceptos y relaciones para una navegación por la estructura más intuitiva que por un listado o una jerarquía.

Por ser una norma relativamente joven no está aún totalmente explorado todo su potencial aplicativo pero, en términos generales, puede considerarse que las desarrolladas hasta el momento se aglutinan en tres grupos: organización y clasificación de recursos, sistemas de navegación y búsqueda y recuperación de información ${ }^{40}$. Cualquiera de estas opciones puede implementarse sola o en conjunto con otras, lo que ha propiciado que sus primeras implantaciones, y las más difundidas, hayan sido en el entorno Web para el diseño de portales o $\operatorname{sitios}^{41}$, intranets o aplicaciones empresariales de gestión (englobados bajo la denominación de Sistemas de Organización del Conocimiento) bien estructurados que pueden ser mantenidos con facilidad.

Además de permitir una organización documental más rica en detalle y relaciones, con visualización y navegación al tiempo, el modelo permite no sólo integrar recursos de distintas fuentes sino tener diferentes perspectivas de la misma información. Así, pueden utilizarse para desarrollar interfaces de ayuda a la navegación y búsqueda de recursos con diversos grados de sofisticación y segmentar distintos perfiles o puntos de vista en función del usuario (AHMED, 2000).

Como mecanismo de recuperación de información permite desde filtrados sencillos mediante el contexto (scope) o la definición de clases de topics, associations y occurrecces, como ya vimos, hasta sistemas más elaborados como consultas complejas, consultas a texto completo y consultas en lenguaje natural (GARSHOL, 2004).

${ }^{40}$ Es de destacar que algunos investigadores del campo de la IA los consideran una herramienta para almacenar e interrogar ontologías (GÓMEZ PÉREZ; BENJAMINS, 2002) y otros, sin embargo, los incluyen como lenguaje para la web semántica que permite describir estructuras de conocimiento (NOY et al., 2001), realzando así el uso que más les interesa.

${ }^{41}$ Ejemplos práctico de aplicación a un sitio web vienen descritos en (OGIEVETSKY; BADGER, 2003) y (PEPPER; GARSHOL, 2003). 


\section{ARTIGO}

Las posibilidades, así, son muy extensas, desde la simple aplicación a la navegación por índices, glosarios, diccionarios, enciclopedias o tesauros, pasando por las bases de datos relacionales ${ }^{42}$ (GRAAUW, 2003), hasta sistemas complejos de gestión documental ${ }^{43}$.

De manera global puede decirse que permiten cualquier aplicación, centrada en el usuario o no, integrando, si así se desea, organización, visualización, navegación, búsqueda y recuperación de información, en cualquier formato y nivel de estructuración, pivotando alrededor de su contenido o significación conceptual. Asimismo, las áreas de aplicación también son muy extensas: edición, gestión empresarial, medicina, bibliotecas digitales y tradicionales, administración, comercio electrónico, educación,... En nuestro campo, de hecho, sus aplicaciones más desarrolladas se concentran en las bibliotecas digitales y en los catálogos en línea, de forma invisible al usuario, combinando, en general, varias de las posibilidades descritas.

\section{BIBLIOGRAFÍA}

AHMED, K. Topic maps for repositories. In: CONFERENCE XML EUROPE, 2000, Paris. Proceedings... $\quad$ Paris, 2000.2 Disponível em: <http://www.gca.org/papers/xmleurope2000/papers/s29-04.html>. Acesso em: 17 jun. 2004.

Topic maps: a practical introduction with case studies. 2001. Disponível em: <http://www.techquila.com/bcase.html>. Acesso em: 29 maio 2004.

information. 2003. XML-Journal. Disponível em: <http://www.syscon.com/xml/articleprint.cfm?id=507>. Acesso em: 02 ago. 2004.

ALTENBURGER, A. Authoring XTM topic maps. part 1. 2000. Disponível em: <http://topicmaps.it.bond.edu.au/docs/6?style=printable>. Acesso em: 28 abr. 2004.

BIEZUNSKI, M.; HAMON, C. A topic map of this conference's proceedings. In: GCA INTERNATIONAL HYTIME CONFERENCE, 3., 1996, Seattle. Proceedings... Seattle, 1996. Disponível em: <http://www.infoloom.com/lHC96/mb214.htm>. Acesso em: 17 maio 2004.

DIJK, P. V. eXchangeable faceted metadata language. 2002. Disponível em: <http://www.xfml.org/>. Acesso em: 30 out. 2003.

42 La página web de HighWire Press (<http://highwire.stanford.edu/help/hbt>), una división de las Bibliotecas de la Universidad de Stanford que produce versiones on-line de revistas de gran impacto y otros contenidos de literatura clínica e investigación médica, incorpora un applet escrito en java que permite recuperar documentos navegando a través de las materias, previamente transformadas en topic map desde una base de datos.

${ }^{43}$ Dar ejemplos de aplicaciones específicas resultaría prolijo pues cada vez van siendo más numerosas. Para hacerse una idea de algunas ya realizadas con éxito puede consultarse (AHMED, 2001) y los diversos enlaces de la página web del seminario Real World Topic Maps en la dirección electrónica http://www.xmluk.org/public/duxford 2003.html. 
FREESE, E. Using Topic Maps: for the representation, management \& discovery of knowledge. In: CONFERENCE XML EUROPE 2000, Paris. Proceedings... Paris, 2000. Disponível em: Disponível em: <http://www.gca.org/papers/xmleurope2000/papers/s22-01.html>. Acesso em: 17 jun. 2004.

Topic maps and RDF. In: PARK, J.; HUNTING, S. (Ed.). XML topic maps: creating and using topic maps for the web. Boston: Addison-Wesley, 2002. p. 283-326.

GARSHOL, L. M. Tolog: a topic map query language. In: XML EUROPE 2001 CONFERENCE, 2001a, Berlin. Proceedings... Berlin, 2001a. Disponível em: <http://www.ontopia.net/topicmaps/materials/tolog.html >. Acesso em: 13 maio 2004.

. Topic maps, RDF, DAML, OIL: a comparison. In: XML CONFERENCE \& EXPOSITION, 2001, Orlando. Proceedings... Orlando, 2001b. Disponível em: <http://www.idealliance.org/papers/xml2001/papers/html/05-04-04.html>. Acesso em: $12 \mathrm{dez}$. 2003b.

. The linear topic map notation: definition and introduction, version 1.2. Ontopia: A/S, 2002. Disponível em: <http://www.ontopia.net/download/ttm.html>. Acesso em: 13 maio 2004.

Living with topic maps and RDF. 2003. Disponivel em: <http://www.ontopia.net/topicmaps/materials/tmrdf.html>. Acesso em: 12 dez. 2003.

Metadata? thesauri? taxonomies? topic maps!: making sense of it all. Journal of Information Science, vol. 30, n. 4, p. 378-391, 2004. Disponível em: <http://www.ontopia.net/topicmaps/materials/tm-vs-thesauri.html>. Acesso em: 5 dez. 2004.

.; MOORE, G. 2003. The XML topic maps (XTM) syntax 1.1: first committee draft 0304 2003. ISO/IEC JTC 1/SC34 N0398. ISO/IEC JTC 1/SC34. Disponível em: $<$ http://www.isotopicmaps.org/sam/sam-xtm>.

GRAAUW, M. D. Using topic maps to extend relational databases. 2003. Disponível em: <http://www.xml.com/pub/a/2003/03/05/tmrdb.html>. Acesso em: 6 out. 2004.

GRØNMO, G. O. 2000. Creating semantically valid topic maps. In: CONFERENCE XML EUROPE, 2000, Paris. Proceedings... Paris, 2000. Disponível em: <http://www.infoloom.com/gcaconfs/WEB/paris2000/S29-02.HTM>. Acesso em: 17 jun. 2004.

GÓMEZ PÉREZ, A.; BENJAMINS, V. R. (Ed.). 2002. Evaluation of ontology-based tools: ontoweb-SIG3. In: INTERNATIONAL CONFERENCE ON KNOWLEDGE ENGINEERING AND KNOWLEDGE MANAGEMENT, 13. 2002, Espana. Proceedings... Espana, 2002. Disponível em: <http://sunsite.informatik.rwth-aachen.de/Publications/CEUR-WS//Vol-62/>. Acesso em: 16 fev. 2004.

HEMRICH, M.; SCHÄFER, U. 1999. XML based linking concept. In: GCA CONFERENCES, XML 99, 1999, Philadelphia. Proceedings... Philadelphia, 1999. Disponível em: <http://www.infoloom.com/gcaconfs/WEB/philadelphia99/hemrich.HTM>. Acesso em: 18 nov. 2003.

INTERNATIONAL ORGANIZATION FOR STANDARDIZATION. Information technology : SGML applications topic maps, ISO/IEC 13250. Geneva: ISO, 2000. Disponível em: <http://www.infoloom.com/tmstands>.

2003.

Information technology: SGML applications topic maps, ISO/IEC 13250. Geneva: ISO,

INTERNET ENGINEERING TASK FORCE. IETF. Uniform resource identifiers (URI): generic syntax. rfc 2396. 1998. Disponível em: <http://www.ietf.org/rfc/rfc2396.txt>. Acesso em: 13 jun. 2004. 
KAMEL BOULOS, M. N.; ROUDSARI, A. V.; CARSON, E. R. Towards a semantic medical Web: healthcybermap's tool for building an RDF metadata base of health information resources based on the qualified dublin core metadata set. Medical Science Monitor, v. 8, n. 7, p. 124-136, 2002. Disponível em: <http://www.protege.stanford.edu/ontologies/dublincore/ hcm_dc_in_protege_newcastle.pdf>. Acesso em: 19 nov. 2003.

KAMINSKY, P. Integrating information on the semantic web using partially ordered multi hypersets. 2002. Tese (Doutorado)-University of Victoria. Acesso em: 10 dez. 2003.

LACHER, M. S.; DECKER, S. On the integration of topic maps and RDF data. In: INTERNATIONAL SEMANTIC WEB WORKING SYMPOSIUM, 2001, Standford, California Disponível em: <http://www.semanticweb.org/SWWS/program/full/paper53.pdf>.

MOORE, G. D. 2001. RDF and TopicMaps: an exercise in convergence. In: XML EUROPE, 2001, Berlin. Disponível em: <http://xml.coverpages.org/moore-topicmapsrdf200105.pdf>. Acesso em: 13 set. 2004.

MOREIRO GONZÁLEZ, J. A.; SÁNCHEZ CUADRADO, S.; MORATO LARA, J. 2003. Panorámica y tendencias en topic maps. Hipertext.net, n. 1, 2003. Disponível em: <http://www.hipertext.net/web/pag182.htm>. Acesso em: 21 jul. 2003.

MUGNAINI, L. Mapping Topic Maps on Relational Databases. [19--?] Disponível em: <http://www.geocities.com/xtopicmaps/mapping_xtm_on_databases.html>. Acesso em: 6 out. 2004.

NOY, N. F. et al. Creating semantic web contents with protege-2000. IEEE Intelligent Systems, v. 16, n. 2, p. 60-71, 2001.

OBRST, L.; LIU, H. Knowledge representation, ontological engineering, and topics maps. In: PARK, J.; HUNTING, S. (Ed.). XML topic maps: creating and using topic maps for the web. Boston: Addison-Wesley, 2002. p. 103-148.

OGIEVETSKY, N. XLST stylesheets for converting ISO 13250 topic map documents into XTM 1.0 syntax. [19--?]. Disponível em: <http:/ www. cogx.com/xslt4tm2xtm.htlm>. Acesso em: 13 maio 2004.

.; BADGER, T. Topic map solutions for kodak digital camera accessories. In: POWERING THE INFORMATION SOCIETY. CONFERENCE XML EUROPE, 2003, Londres. Proceedings... Londres, $2003 . \quad$ Disponível em: <http://www.idealliance.org/papers/dx_xmle03/papers/02-03-02/02-03-02.html>.

PEPPER, S. Euler, topic maps, and revolution. In: CONFERENCE XML EUROPE, 1999, Granada. Proceedings... Granada, 1999. Disponível em: <http://www.infoloom.com/tmsample/pep4.htm>. Acesso em: 12 set. 2003.

The TAO of topic maps: finding the way in the age of infoglut. 2002. Disponível em: <http://www.ontopia.net/topicmaps/materials/tao.html>. Revisión del original de 2000. Acesso em: 12 set. 2003.

. Ten theses on topic maps and RDF. Disponível em: <http://www.ontopia.net/topicmaps/materials/rdf.html>. Acesso em: 17 maio 2004.

2004. Towards seamless knowledge: integrating public sector portals. In: XML 2004 CONFERENCE \& EXPOSITION, 2004, Washington. Disponível em: $<$ Towards\%20Seamless\%20Knowledge[1]>. Acesso em: 6 dez. 2004. 


\section{ARTIGO}

PEPPER, S.; GARSHOL, L. M. The XML papers: lessons on applying topic maps. In: POWERING THE INFORMATION SOCIETY. CONFERENCE XML EUROPE, 2003, Londres. Proceedings ... Disponível em: <http://www.idealliance.org/papers/dx_xmle03/papers/04-0301/04-03-01.html>.

; GRONMO, G. O. Towards a general theory of scope. In: EXTREME MARKUP LANGUAGES, 2001, Montréal. Proceedings... Montréal, 2001. Disponível em: <http://www.ontopia.net/topicmaps/materials/scope.htm>. Acesso em: 27 mar. 2004.

RATH, H. H. White paper: the topic map handbook. Gütersloh: $\mathrm{GmbH}, 2003$. Disponível em: <http://www.empolis.com/download/docs/whitepapers/empolistopicmapswhitepaper_eng.pdf>. Acesso em: 17 dez. 2003.

SIGEL M. A. A. Towards knowledge organization with topic maps. In: CONFERENCE XML EUROPE, 2000, Paris. Proceedings... Paris, 2000. Disponível em: <http://www.gca.org/papers/xmleurope2000/papers/s22-02.html>. Acesso em: 17 jun. 2004.

TOPICMAPS. XML topic maps (XTM) 1.0 specification. In: PEPPER, S.; MOORE, G. (Ed.). TopicMaps.Org. 2001. Disponível em: <http://www.topicmaps.org/xtm/1.0>. Acesso em: 10 set. 2003.

VATANT, B. Cooking for the semantic web: OWL and topic map pudding. 2003. Disponível em: <http://www.mondeca.com/owl/owltm.htm>. Acesso em: 15 set. 2003.

Ontology-driven topic maps. In: XML EUROPE, 2004, Amsterdam. Disponível em: <http://www.idealliance.org/europe/04/call/xmlpapers/03-03-03.91/.03-03-03.html>. Acesso em: 15 maio 2004.

Maria Jesús Colmenero Ruiz

Becaria de Investigación del Departamento de Biblioteconomía y Documentación, Universidad Carlos III de Madrid E-mail: mcolmene@bib.uc3m.es

Artigo aceito para publicação em: 07/2005 